\title{
Collagen I induction by high glucose levels is mediated by epidermal growth factor receptor and phosphoinositide 3-kinase/Akt signalling in mesangial cells
}

\author{
D. Wu $\cdot$ F. Peng $\cdot$ B. Zhang $\cdot$ A. J. Ingram $\cdot$ B. Gao • \\ J. C. Krepinsky
}

Received: 13 November 2006 / Accepted: 3 May 2007 / Published online: 11 July 2007

(C) Springer-Verlag 2007

\begin{abstract}
Aims/hypothesis Glomerular matrix accumulation is a hallmark of diabetic nephropathy. Recent data have linked the serine/threonine kinase protein kinase B (Akt) to matrix modulation. Here, we studied its role in high glucoseinduced collagen elaboration by mesangial cells.

Methods Primary rat mesangial cells were treated with high glucose levels $(30 \mathrm{mmol} / \mathrm{l})$ or mannitol as osmotic control. Western blots, northern blots, ELISA and immunohistochemistry were used for assessment. Diabetes was induced in rats by streptozotocin.

Results Phosphorylated Akt at S473 (pAktS473), corresponding to Akt activation, was seen in diabetic glomeruli. In mesangial cells, high glucose levels induced pAktS473 by $20 \mathrm{~min}$. This was sustained to $72 \mathrm{~h}$, while mannitol had no effect. Akt activation by kinase assay and phosphorylation on threonine 308 was also observed. Phosphoinositide 3-kinase (PI3K) inhibitors LY294002 (20 $\mu \mathrm{mol} / \mathrm{l})$ and wortmannin $(100 \mathrm{nmol} / \mathrm{l})$ prevented pAktS473. Collagen IA1 transcript and collagen I protein upregulation by high glucose levels were inhibited by PI3K blockade, as was collagen I secretion into the medium (ELISA). Dominant-negative Akt overexpression also
\end{abstract}

Electronic supplementary material The online version of this article (doi:10.1007/s00125-007-0721-1) contains supplementary material, which is available to authorised users.

D. Wu $\cdot$ F. Peng $\cdot$ B. Zhang $\cdot$ A. J. Ingram $\cdot$ B. Gao

J. C. Krepinsky

Division of Nephrology, McMaster University,

Hamilton, ON, Canada

J. C. Krepinsky $(\square)$

McMaster University, St Joseph's Hospital Site,

50 Charlton Ave E, Rm T3311,

Hamilton, ON L8N 4A6, Canada

e-mail: krepinj@mcmaster.ca inhibited high glucose-induced collagen IA1 transcript and collagen I protein production. Since signalling through the epidermal growth factor receptor (EGFR) can activate PI3K-Akt, we studied its activation by high glucose levels. EGFR was correspondingly activated by $10 \mathrm{~min}$; mannitol had no effect. EGFR activation was also seen in glomeruli from diabetic rats and co-localised with collagen IA1 in diabetic glomeruli. Specific EGFR inhibition (AG1478, $5 \mu \mathrm{mol} / 1$ or dominant-negative EGFR) blocked high glucose-induced pAktS473, phosphorylation on threonine 308 and activation of the EGFR downstream target p44 extracellular signal-regulated kinase (Erk) mitogen-activated protein kinase. Finally, EGFR inhibition also blocked high glucose-induced collagen I upregulation at transcriptional and protein levels.

Conclusions/interpretation We conclude that EGFR-PI3KAkt signalling mediates high glucose-induced collagen I upregulation in mesangial cells and that this pathway is activated in diabetic glomeruli. Targeting its components may provide a new therapeutic approach to diabetic kidney disease.

Keywords Akt · Collagen - Diabetic nephropathy ·

Epidermal growth factor receptor $\cdot$ Extracellular matrix $\cdot$ High glucose $\cdot$ Mesangial cell $\cdot$ Phosphoinositide 3 -kinase $\cdot$ Protein kinase $\mathrm{B} \cdot$ Transactivation

$\begin{array}{ll}\text { Abbreviations } \\ \text { Akt } & \text { protein kinase B } \\ \text { AP-1 } & \text { activator protein 1 } \\ \text { EGF } & \text { epidermal growth factor } \\ \text { EGFR } & \begin{array}{l}\text { epidermal growth factor receptor } \\ \text { Erk }\end{array} \\ \text { extracellular signal-regulated kinase } \\ \text { GSK-3 } & \begin{array}{l}\text { glycogen synthase kinase-3 } \\ \text { mitogen-activated protein kinase }\end{array}\end{array}$


pAktS473 phosphorylated Akt at Ser473

PDGFR platelet-derived growth factor receptor

PI3K phosphoinositide 3-kinase

Sp1 Sp1 transcription factor

\section{Introduction}

The kidney is a major site of diabetic microvascular complications and glomerular matrix accumulation is the pathological hallmark of diabetic nephropathy [1]. Hyperglycaemia is the primary pathogenetic factor in diabetic renal disease [2]. Maintenance of normoglycaemia or interruption of angiotensin II signalling, current mainstays of therapy, can at best only delay onset of renal failure [2, 3]. Consequently, there is a need to identify new therapeutic strategies for diabetic nephropathy.

Under high glucose conditions, mesangial cells synthesise extracellular matrix proteins including collagen, the production of which is increased in diabetic glomeruli in humans and animal models [4]. Recent data from our group and others indicate that the serine-threonine kinase protein kinase B (Akt) may play a role in matrix elaboration [5-8]. Furthermore, Akt has been implicated in collagen I upregulation in lung fibroblasts and mesangial cells exposed to high doses of TGF- $\beta$, a profibrotic cytokine thought to play a central role in diabetic glomerular sclerosis $[8,9]$.

Akt is a serine-threonine kinase important in cell processes such as proliferation, survival and metabolism [10]. Phosphoinositide 3-kinase (PI3K) is a well-established upstream mediator of Akt activation, generating phosphorylated lipid second messengers, that recruit proteins with pleckstrin homology domains, such as Akt, to the membrane [11], where Akt is then activated by phosphorylation at both T308 and S473. The former is effected by phosphoinositide-dependent protein kinase 1 [11]. The identity of the S473 kinase is not definitively known. Both phosphorylation events are required for activation, although that of S473 (pS473) is known to parallel full PI3K/Akt activation [12]. After activation, Akt mediates the inhibition or activation of a number of downstream effectors, including transcription factors, through serine/threonine phosphorylation events $[10,11]$.

PI3Ks are heterodimeric, comprising catalytic (p110) and regulatory subunits (p85 or p101) [13]. The activation of PI3K itself requires the release of autoinhibition of the catalytic subunit by its regulatory subunit. This occurs when the regulatory subunit interacts with phosphotyrosine residues of activated growth factor receptors, G protein coupled receptors or adaptor proteins [11]. These include the epidermal growth factor (EGF) receptor (EGFR), with EGF stimulation leading to Akt activation [10]. Indirect transactivation of the EGFR by various stimuli, including angiotensin II, oxidative stress and mechanical stress, can also result in Akt activation [5, 14].

In this study, we show that Akt is activated in diabetic glomeruli and that high glucose conditions lead to both short- and longer-term Akt activation in glomerular mesangial cells. The activation of PI3K/Akt signalling through transactivation of the EGFR mediates collagen I production in mesangial cells in a hyperglycaemic environment. Our data suggest a novel role for EGFR/Akt signalling in diabetes.

\section{Methods}

Animal studies Experiments were performed with male Sprague-Dawley rats weighing 200 to $225 \mathrm{~g}$ (Charles River, Montreal, QC, Canada) in accordance with Canadian Council on Animal Care guidelines. Diabetes was induced with $60 \mathrm{mg} / \mathrm{kg}$ streptozotocin injection by tail vein. Control rats received equal volume of $0.1 \mathrm{~mol} / 1$ citrate buffer, $\mathrm{pH}$ 4.5. Hyperglycaemia (blood glucose $>17 \mathrm{mmol} / \mathrm{l}$ ) was confirmed 3 days after injection (Precision Xtra; Abbott Laboratories, Medisense Products, Bedford, MA, USA). All rats had free access to regular lab chow and water. After 2 or 4 weeks $(n=4$ and $n=6$ per group per condition respectively), weight and serum glucose were obtained. Rats were then anaesthetised for kidney removal. A small cortical section was placed into optimal cutting temperature (OCT) compound for immunohistochemistry. Cortex from individual rats was then differentially sieved for glomeruli [5]. Protein was extracted from isolated glomeruli ( $>95 \%$ purity by light microscopy) as outlined below.

Cell culture Sprague-Dawley primary rat mesangial cells (passages 6-15) were cultured in DMEM supplemented with 20\% fetal calf serum (Invitrogen, Burlington, ON, Canada), streptomycin $(100 \mu \mathrm{g} / \mathrm{ml})$ and penicillin (100 units $/ \mathrm{ml}$ ) at $37^{\circ} \mathrm{C}$ in $95 \%$ air, $5 \% \mathrm{CO}_{2}$. Medium contained $5.6 \mathrm{mmol} / \mathrm{l}$ glucose. Either $24.4 \mathrm{mmol} / \mathrm{l}$ glucose (final concentration $30 \mathrm{mmol} / \mathrm{l}$ ) or mannitol was added for high glucose levels or osmotic control respectively. Mesangial cells were made quiescent by serum deprivation for $24 \mathrm{~h}$ prior to treatment. Medium was changed every 2 days for experiments of longer duration. Pharmacological inhibitors were added prior to high glucose as follows: wortmannin (Sigma, Oakville, ON, Canada) $100 \mathrm{nmol} / 1$ for $60 \mathrm{~min}$; LY294002 (Sigma) $20 \mu \mathrm{mol} / \mathrm{l}$ for $30 \mathrm{~min}$; AG1478 (Sigma) $5 \mu \mathrm{mol} / 1$ for $30 \mathrm{~min}$, AG1295 (Sigma) $5 \mu \mathrm{mol} / \mathrm{l}$ for $30 \mathrm{~min}$.

Protein extraction and western immunoblotting Cells were lysed and protein extracted as previously described [15]. Briefly, cells were lysed in a buffer containing $20 \mathrm{mmol} / \mathrm{l}$ 
Tris- $\mathrm{HCl}(\mathrm{pH} 7.5), 150 \mathrm{mmol} / 1 \mathrm{NaCl}, 1 \%$ Triton X-100, $1 \mathrm{mmol} / 1 \mathrm{EDTA}, 1 \mathrm{mmol} / 1 \mathrm{EGTA}, 2.5 \mathrm{mmol} / 1$ sodium pyrophosphate, $1 \mathrm{mmol} / 1 \quad \beta$-glycerophosphate, $1 \mathrm{mmol} / 1$ sodium vanadate, $1 \mathrm{mmol} / \mathrm{l}$ phenylmethylsulfonyl fluoride, $1 \mu \mathrm{g} / \mathrm{ml}$ leupeptin and $2 \mu \mathrm{g} / \mathrm{ml}$ aprotinin. Cell lysates were centrifuged at $4^{\circ} \mathrm{C}$ and $16,200 \mathrm{~g}$ for $10 \mathrm{~min}$ to pellet cell debris. The supernatant fraction $(50 \mu \mathrm{g})$ was separated on $10 \%$ SDS-PAGE and western blotting performed as described [15]. Antibodies used were: polyclonal phosphoAkt S473 (1:1000), polyclonal phospho-Akt T308 (1:1000), polyclonal total Akt (1:1000), polyclonal phospho-p44/42 mitogen-activated protein kinase (MAPK) $(1: 1000)$, polyclonal total p44/42 MAPK (1:1000), polyclonal phosphoEGFR Y1068 (1:1000) (all Cell Signaling, Boston, MA, USA); and monoclonal $\beta$-actin (1:5000; Sigma).

Akt activity assay Protein was extracted as described above and Akt activity assay performed according to product specifications (Cell Signaling). Briefly, total Akt was immunoprecipitated from $200 \mu \mathrm{g}$ of cell lysate. After washing, kinase reactions were carried out for $30 \mathrm{~min}$ at $30^{\circ} \mathrm{C}$ in kinase buffer containing $200 \mu \mathrm{mol} / \mathrm{l}$ ATP and $1 \mu \mathrm{g}$ glycogen synthase kinase-3 (GSK-3) fusion protein. Beads were resuspended in $2 \times$ reducing sample buffer, boiled for $5 \mathrm{~min}$ and the supernatant fractions resolved on 10\% SDS-PAGE. Membranes were probed sequentially with rabbit polyclonal phospho-GSK-3 $\alpha / \beta$ Ser 21/9 (1:1000; Cell Signaling) and total Akt to ensure equal immunoprecipitation.

Northern blot analysis Total RNA (10 $\mu \mathrm{g})$, extracted using Trizol (Invitrogen), was separated on a formaldehydeagarose gel and transferred to a nylon membrane (Hybond; Amersham Biosciences, Baie d'Urie, QC, Canada). Hybridisation was performed with ${ }^{32} \mathrm{P}$-labelled dCTP-randomprimed cDNA probes prepared from collagen IA1 or $\beta$-actin cDNA amplified by PCR.

Enzyme-linked immunosorbent assay After treatment, conditioned media were collected, debris removed by lowspeed centrifugation at $1,300 \mathrm{~g}$ and plates coated at $4{ }^{\circ} \mathrm{C}$ overnight with media (1:3 dilution) in ELISA coating buffer (Sigma), followed by blocking and incubation with monoclonal anti-collagen I (Sigma, 1:2000) in blocking buffer as previously published [15]. After incubation with alkaline phosphatase-conjugated secondary antibody (1:30,000; Sigma), $p$-nitrophenyl phosphate was added and reactions read at $405 \mathrm{~nm}$ in a microplate autoreader.

Mesangial cell infection Epitope-tagged dominant-negative Akt (HA-AktAAA, kindly provided by J. Woodgett [16], Samuel Lunenfeld Research Institute, University of Toronto, Toronto, ON, Canada) or dominant-negative EGFR (dnEGFR, kindly provided by S. Parsons, University of
Virginia Health System, Charlottesville, VA, USA) was cloned into the empty vector pLHCX (Clontech, Mountain View, CA, USA) for retroviral infection and mesangial cells infected as previously described [5]. Competent virus capable of single infection was generated using the vesicular stomatitis virus system (Stratagene, La Jolla, CA, USA) and mesangial cells, passages 5 to 12 , were exposed to virus concentrated by centrifugation for $90 \mathrm{~min}$ at $50,000 \mathrm{~g}, 4^{\circ} \mathrm{C}$ in the presence of polybrene. Seventy-two hours after infection a 2 week antibiotic selection period was begun. Experiments were performed using a population of pooled, stably infected mesangial cells.

Immunohistochemistry Renal cortical cryosections $(6 \mu \mathrm{m})$ were air-dried, fixed in ice-cold $100 \%$ methanol for $15 \mathrm{~min}$, washed and permeabilised with $0.2 \%$ Triton $\mathrm{X}-100$ for $15 \mathrm{~min}$. After further washes, sections were blocked with $5 \%$ donkey serum $/ 0.2 \%$ Triton $\mathrm{X}-100$ in PBS and incubated with primary antibodies overnight at $4{ }^{\circ} \mathrm{C}$. Antisera used were: rabbit polyclonal anti-pAktS473 (1:50); rabbit polyclonal anti-pEGFR Y1068 (both Cell Signaling); sheep polyclonal anti-von Willebrand Factor $(2 \mu \mathrm{g} / \mathrm{ml}$; Cedarlane, Burlington, ON, Canada); polyclonal anti-total EGFR (1:100; Cell Signaling); and monoclonal anti-collagen type I (1:100; Sigma). After washing, FITC- and rhodamineconjugated secondary antibodies $(1: 100$; Jackson ImmunoResearch, West Grove, PA, USA) were applied for $1 \mathrm{~h}$ and sections mounted (Vectashield, Vector Laboratories [Canada], Burlington, ON, Canada) after a final wash. Images were acquired with a Zeiss fluorescent microscope (Carl Zeiss Canada, Toronto, ON, Canada).

Statistical analyses For experiments with more than two groups, statistical analyses were performed using one-way ANOVA, with Tukey's honestly significant difference (HSD) test used for post-hoc analysis. A $t$ test was used for experiments with only two groups. A $p$ value $<0.05$ (two-tailed) was considered significant. Data are presented as the mean \pm SEM. The number of repetitions for experiments is given in figure legends $(n)$. SPSS for Windows 11.0 (SPSS, Chicago, IL, USA) was used for analysis.

\section{Results}

Akt is activated in diabetic glomeruli Akt has been linked to the production of matrix proteins and with its characteristic matrix accumulation, glomerular sclerosis is the pathological hallmark of diabetic nephropathy [1]. We first sought to determine whether Akt was activated in the diabetic setting in a relevant in vivo model, streptozotocininduced type 1 diabetes. Diabetic rats had significant 
hyperglycaemia compared with controls at both 2 and 4 weeks $(27.5 \pm 1.3$ vs $6.1 \pm 1.1 \mathrm{mmol} / 1, p<0.001)$. Both cortex and glomeruli were analysed for Akt activation, as assessed by immunoblotting for phospho-Ser 473 . Figure 1a,b shows that Akt was activated in whole cortex after 2 weeks of diabetes and that this was magnified in glomerular isolates. Similar activation of Akt was observed after 4 weeks of diabetes (Fig. 1c,d). To further confirm activation of Akt in diabetic glomeruli, we used immunohistochemistry to identify S473-phosphorylated Akt in cortical sections. As seen in Fig. 1e, S473 phosphorylation (green) was increased at 4 weeks after induction of diabetes. Since glomeruli consist of a rich capillary network anchored by the mesangium, we used the endothelial cell marker von Willebrand factor (red) to confirm glomerular localisation of immunofluorescence of phosphorylated Akt at Ser473 (pAktS473) [17].

High glucose levels induce Akt activation in cultured mesangial cells We then tested whether high glucose levels could activate Akt in vitro. Mesangial cells were exposed to $30 \mathrm{mmol} / \mathrm{l}$ glucose for varying times and Akt S473 phosphorylation assessed by immunoblotting. Akt phosphorylation was seen by $20 \mathrm{~min}$ and sustained with prolonged incubation up to $72 \mathrm{~h}$ (Fig. 2a-d). Phosphorylation on threonine 308 is also required for full activity [18]; high glucose-induced phosphorylation on threonine 308 was indeed observed in mesangial cells (Fig. 2e). To confirm that phosphorylation on S473 correlated with Akt activity [12], we performed an Akt activity assay. As expected, high glucose level induced a significant increase in phosphorylation of the substrate GSK-3 by immunoprecipitated total Akt, reaching statistical significance at 20 min, see ESM Fig. 1a,b. A sustained increase in Akt activity was seen up to $24 \mathrm{~h}$ (data not shown). To ensure these effects were not osmotically mediated, we incubated mesangial cells with equivalent concentrations of mannitol. No effects on pAktS473 or phosphorylation on threonine 308 were observed at 1 or $24 \mathrm{~h}$ of mannitol treatment (ESM Fig. 1c; Fig. 2e).

Since PI3K is a well-established upstream mediator of Akt activation [10, 19], we next examined the effects of two inhibitors of this enzyme on high glucose-induced Akt activation. Wortmannin irreversibly inhibits PI3K activity by covalently binding to the p110 catalytic subunit, whereas LY294002 is a reversible inhibitor, binding to the ATP-binding pocket of the catalytic subunit $[8,10]$. Both of these inhibitors effectively blocked Akt phosphorylation after incubation at high glucose levels for $1 \mathrm{~h}$, with no baseline effects observed (ESM Fig. 2a,b).

High glucose-induced collagen production requires Akt activation Overexpression of constitutively active Akt increases collagen I production in hepatic stellate cells [7] and collagen IV production in embryoid bodies [6]. We have shown Akt activation to be important in the elaboration of collagen I in response to mechanical strain [5]. Since accumulation of extracellular glomerular matrix is the pathogenic hallmark of diabetic renal disease, we studied whether Akt activation was important in collagen production a

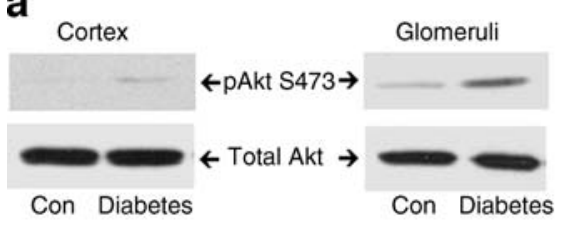

b

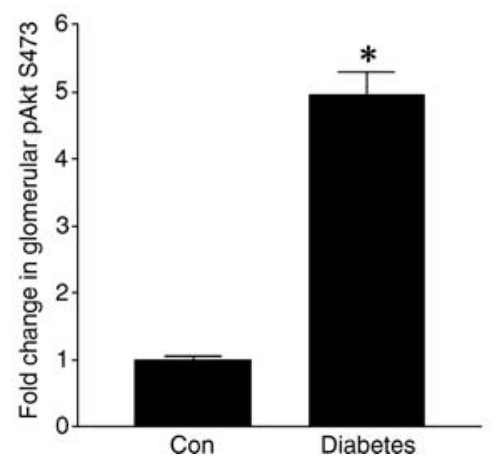

C

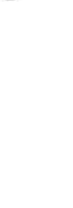

d

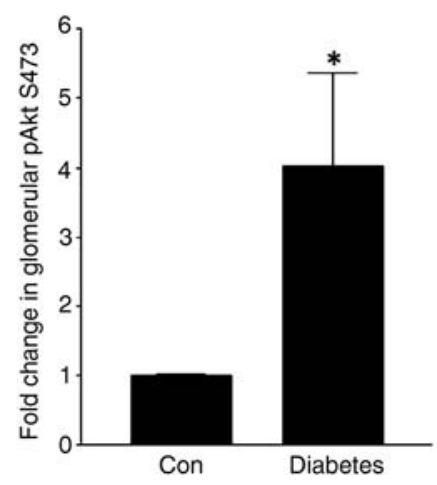

e

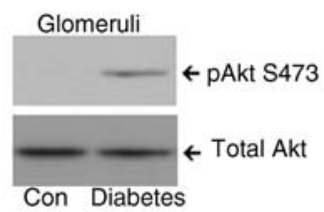

Con
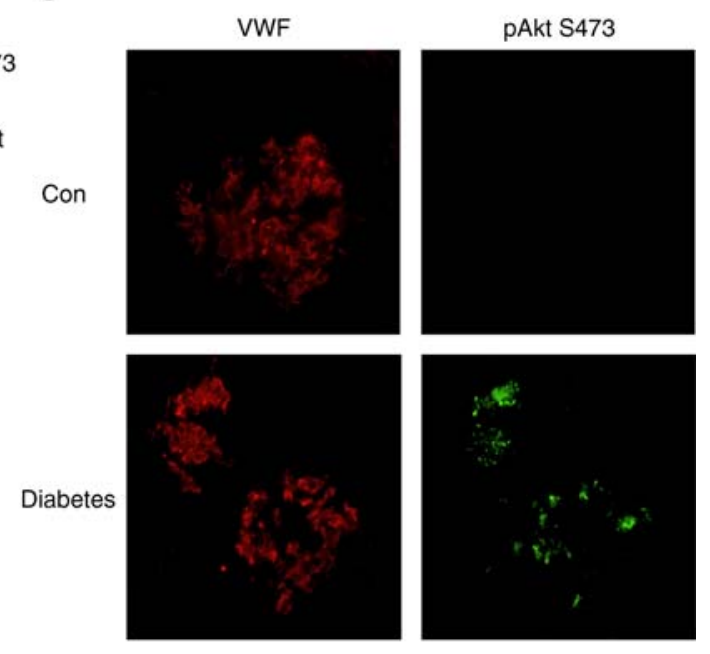

Fig. 1 Akt is activated in diabetic glomeruli. After 2 (a, b) and 4 weeks (c, d), increased AktS473 phosphorylation was seen in isolated glomeruli from streptozotocin-induced diabetic rats. At 2 weeks, this was more pronounced than pAktS473 in whole cortex. (b) $0.05(\mathbf{b}) ; n=4(\mathbf{b}) ; n=6(\mathbf{d})$. Fifty micrograms of protein were used for cortex and glomerular immunoblots. e Immunofluorescence shows increased AktS473 phosphorylation (green) in diabetic glomeruli, identified by von Willebrand factor (red). Con control 
a
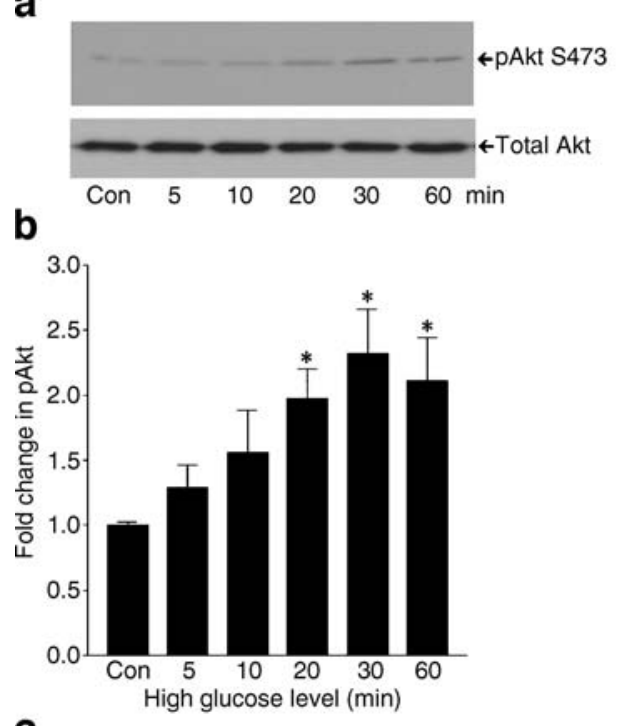

C

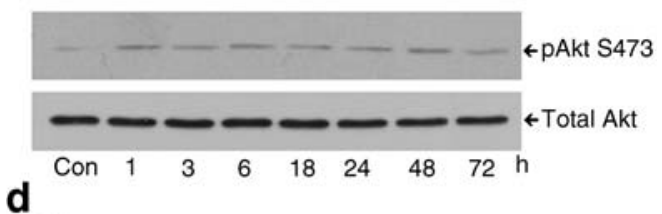

d
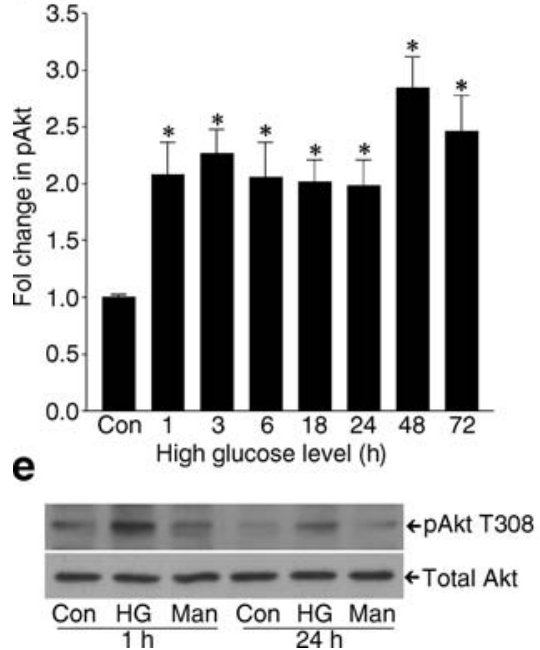

Fig. 2 High glucose levels activate Akt in mesangial cells. a Immunoblotting showed a time-dependent increase in pAktS473, which became significant(b) by $20 \mathrm{~min}$ of high glucose levels; ${ }^{*} p<0.05$ vs control (Con), $n=5$. This increase was sustained (c, d) up to $72 \mathrm{~h}$; ${ }^{*} p<0.05$ vs control, $n=8$. e Akt threonine 308 phosphorylation was also observed in mesangial cells in response to both 1 and $24 \mathrm{~h}$ of high glucose levels $(H G)$, as shown by immunoblotting. Mannitol (Man) was without effect $(n=2)$

by mesangial cells in response to high glucose levels. We first established a time course for collagen I induction by high glucose levels. Collagen IA1 mRNA was determined by northern blotting after exposure of mesangial cells to high glucose levels for 1 to 4 days as shown in ESM Fig. 3a, with maximal response seen at 3 days. A similar induction in cellular collagen I was seen by immunoblotting (ESM
Fig. 3b). In subsequent studies, we thus used 3 days of high glucose incubation to assess collagen I production.

We next assessed whether PI3K/Akt signalling mediates high glucose-induced collagen I production. Mesangial cells were incubated with high glucose levels in the presence or absence of wortmannin $(100 \mathrm{nmol} / \mathrm{l})$ or LY294004 (20 $\mu \mathrm{mol} / 1)$. Both PI3K inhibitors completely abrogated the collagen I upregulation induced by high glucose levels at both mRNA (Fig. 3a,b) and protein (Fig. 3c,d) levels. No toxic effects were observed with either inhibitor. To ensure that collagen I cellular upregulation correlated with enhanced collagen I protein secretion into the medium, we performed ELISA on conditioned medium. High glucose levels increased collagen I secretion by mesangial cells and both PI3K inhibitors prevented this (Fig. 3e). Equivalent incubation of mesangial cells with mannitol did not alter collagen IA1 transcript levels or collagen I protein expression and secretion, as seen in Fig. 3a-e.

To confirm that Akt activation is required for high glucose-induced collagen I upregulation, we studied responses in cells overexpressing the dominant-negative Akt mutant AktAAA. We have previously shown that stable overexpression of this mutant in mesangial cells prevented signalling downstream of Akt as assessed by phosphorylation of the Akt substrate GSK-3 [5]. In Fig. 4a-d, mesangial cells retrovirally infected with the empty vector pLHCX showed the expected increase in collagen IA1 transcript and collagen I protein levels in response to high glucose levels. This increase at both the transcript and protein levels was completely inhibited in cells expressing dominant-negative Akt.

Epidermal growth factor receptor is transactivated by high glucose levels and mediates Akt activation EGFR has been shown to serve as an intermediary in the activation of signalling pathways initiated by stimuli other than EGF through a process termed transactivation [20]. Furthermore, the regulatory $\mathrm{p} 85$ subunit of PI3K can interact with growth factor receptors including the EGFR [11]. We thus tested whether EGFR transactivation might play a role in high glucose-induced Akt activation in mesangial cells. We incubated mesangial cells in high glucose levels for varying times and assessed EGFR activation by the status of its autophosphorylation site Y1068 [21]. Figure 5a,b shows that high glucose-induced EGFR activation occurred prior to Akt activation. Moreover, Akt S473 phosphorylation induced by $1 \mathrm{~h}$ of high glucose treatment was completely prevented by the highly-specific EGFR inhibitor AG1478 [22] (Fig. 5c,d), as was T308 phosphorylation (Fig. 5e,f). We verified that AG1478 inhibits EGFR Y1068 phosphorylation by high glucose levels $(30 \mathrm{~min}$ ) and that mannitol was without effect (ESM Fig. 4a). We sought to further specifically implicate EGFR in high glucose-induced 

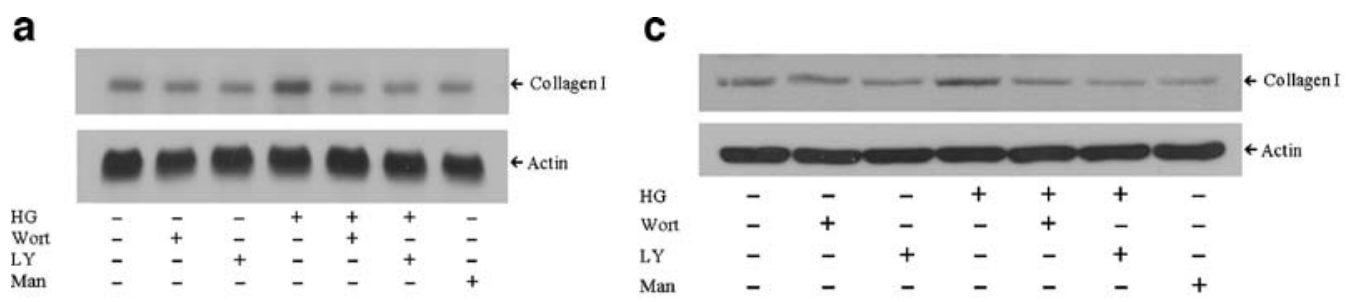

b

d

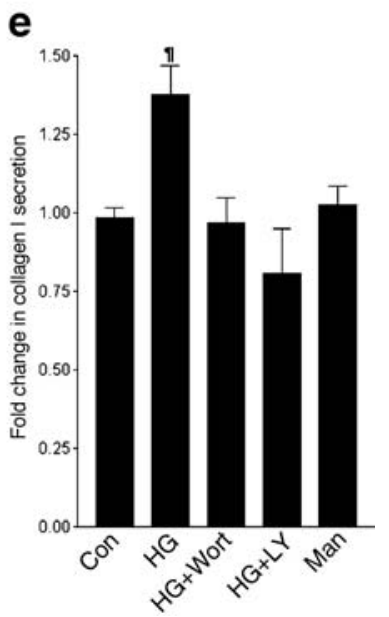

Fig. 3 PI3K inhibition prevents glucose-induced collagen I production. a, b Collagen IA1 mRNA upregulation by 3 days of high glucose $(H G)$ levels was blocked by PI3K inhibitors LY294002 (LY; $20 \mu \mathrm{mol} / \mathrm{l})$ and wortmannin (Wort; $100 \mathrm{nmol} / \mathrm{l}$ ), as assessed by northern blot and quantified in bar graph (b). Data were normalised to actin. ${ }^{* *} p<0.01$ vs all others (b), $n=6$. c Western blot showed that cellular collagen I protein was increased by 3 days of high glucose levels. This was also

pAktS473; thus, we transfected mesangial cells with a dnEGFR and examined Akt S473 phosphorylation in response to high glucose levels by immunoblotting. Figure $5 \mathrm{~g}$ shows effective overexpression of this construct, prevented by $\mathrm{PI} 3 \mathrm{~K}$ inhibition. d Bar graph, quantifying western blot densitometry; ${ }^{\S} p<0.03$ vs all others, $n=5$. e Collagen I secreted into the medium after 3 days of high glucose exposure was detected by ELISA. PI3K inhibitors prevented the increase seen with high glucose levels; ${ }^{\Phi_{p}} p 0.04$ vs all others, $n=5$. All panels show mannitol (Man) to be without effect. Con control

while Fig. 5h,i shows the abrogation of Akt S473 phosphorylation at high glucose levels by dnEGFR.

We have previously shown high glucose-induced p44/42 extracellular signal-regulated kinase (Erk) MAPK activa-
Fig. 4 Dominant-negative Akt inhibits high glucose-induced collagen I production. A pooled population of stably infected mesangial cells with dominantnegative (dn) Akt (HA-AktAAA) or empty vector $\mathrm{pLHCX}$ was studied. a Northern blot showed that high glucose-induced collagen IA1 transcript upregulation was seen only in mesangial cells with empty vector. b Graph quantifying results of blot (a). Closed bar pLHCX; dotted bar dnAkt. ${ }^{*} p<0.05$ vs control (Con) for pLHCX, $n=3$. c, d Similarly, collagen I protein upregulation by high glucose levels was also absent in AktAAA mesangial cells, ${ }^{\natural} p<0.04$ vs con for pLHCX, $n=7) . d$, day

\section{a}
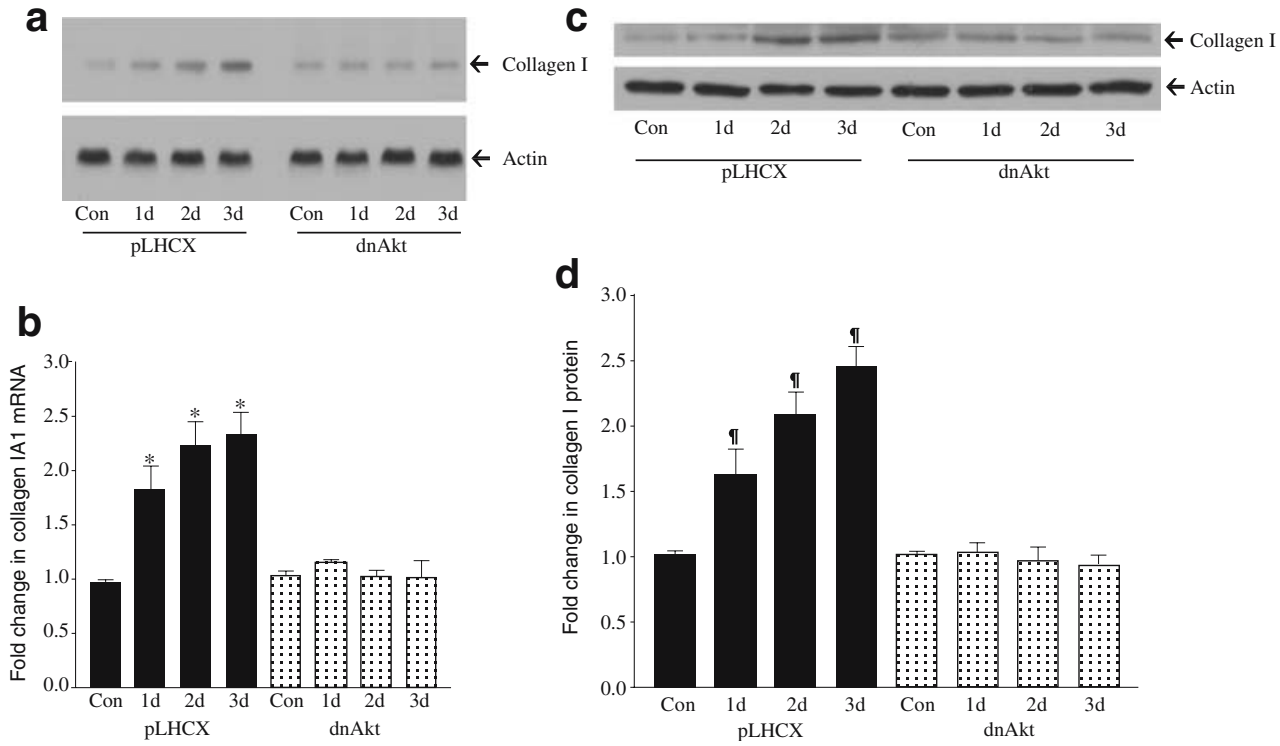

d

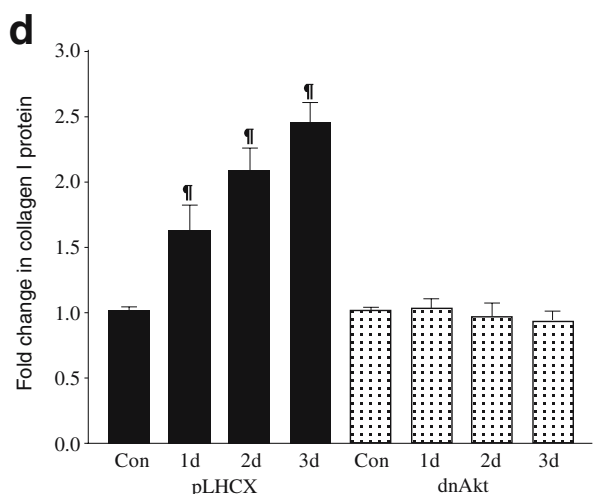


a

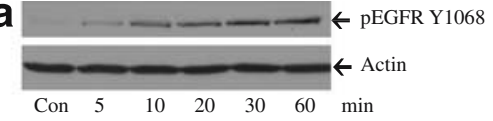

b

b

C
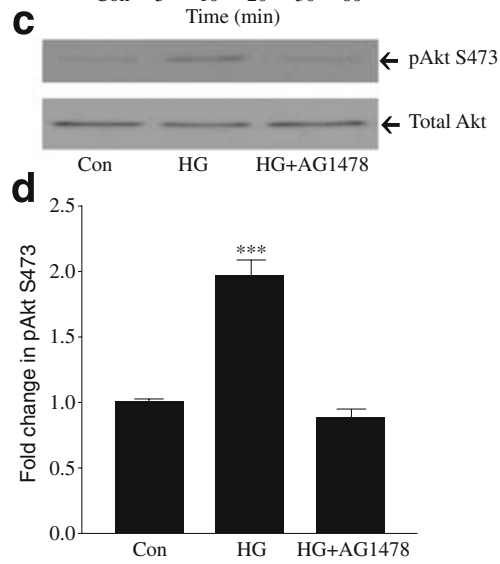

e
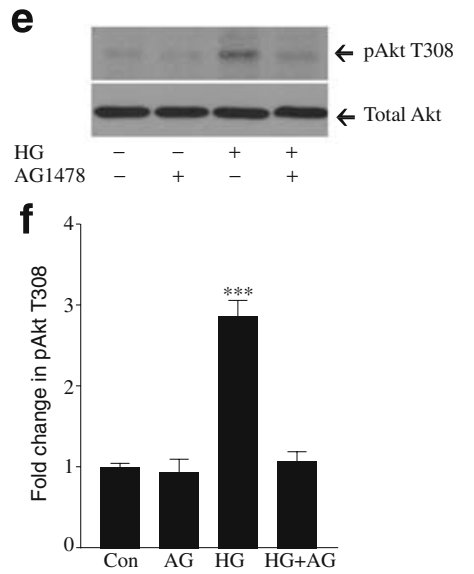

g

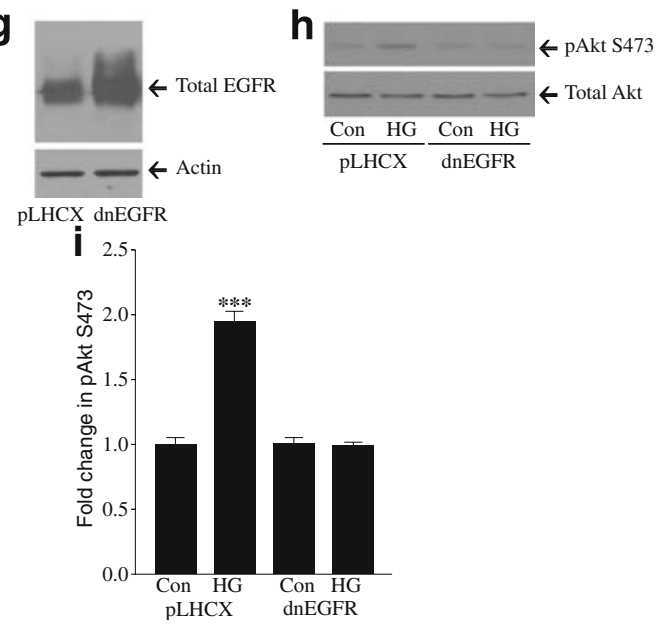

4Fig. 5 Transactivation of epidermal growth factor receptor mediates Akt activation. a EGFR autophosphorylation at Y1068 (indicative of activation) was assessed by immunoblotting and was significantly increased (b) by high glucose (HG) levels, ${ }^{*} p<0.05$ vs control (Con), $n=5$. c EGFR inhibition with AG1478 ( $5 \mu \mathrm{mol} / 1,30 \mathrm{~min})$ prevented high glucose-induced pAktS473, showing dependence on EGFR transactivation. d Bar graph quantifying blot (c), $* * * p<0.001$ vs all others, $n=6$. e High glucose-induced Akt phosphorylation on T308 at $1 \mathrm{~h}$ was also prevented by the EGFR inhibitor AG1478, as quantified in bar graph (f), $* * * p<0.001$ vs all, $n=4$. $\mathbf{g}$ A pooled population of stably infected mesangial cells expressing dominant-negative (dn) EGFR (K721A) or empty vector pLHCX was generated. Immunoblot shows overproduction of dnEGFR. h Immunoblot showing that high glucose levels for $1 \mathrm{~h}$ did not induce Akt S473 phosphorylation in cells with dominant-negative EGFR, with results quantified in bar graph (i), $* * * p<0.001$ vs all others, $n=3$

tion in mesangial cells [23]. Others have demonstrated involvement of EGFR in this phenomenon in vascular smooth muscle cells [24]. Thus, we sought to determine whether EGFR was involved in high glucose-induced Erk activation in mesangial cells. Immunoblotting revealed increased Erk phosphorylation on T202/Y204, a widely accepted substitute for activation in response to high glucose levels, while also showing that this was prevented by EGFR inhibition (ESM Fig. 4b,c).

High glucose-induced collagen production requires EGFR transactivation Since we observed that Akt activation is dependent on EGFR transactivation, we studied whether this growth factor played a role in high glucose-induced collagen I upregulation. Mesangial cells were exposed to high glucose levels for 3 days in the presence or absence of the EGFR inhibitor AG1478 (5 $\mu \mathrm{mol} / \mathrm{l})$. Figure $6 \mathrm{a}, \mathrm{b}$ shows that high glucose-induced collagen IA1 transcript upregulation, assessed by northern blotting, was abrogated by inhibition of EGFR activation. Collagen I protein induction by high glucose levels was similarly prevented (Fig. 6c,d). Overexpression of dnEGFR in mesangial cells confirmed that this receptor is required for high glucose-induced collagen IA1 transcript upregulation (Fig. 6e). Plateletderived growth factor receptor (PDGFR) may heterodimerise with EFGR and play a role in EGFR transactivation [25]. However, using the highly specific PDGFR inhibitor AG1295 [22], immunoblotting did not show a role for PDGFR in Akt S473 phosphorylation, and northern blot analysis did not show a role for PDGFR in collagen IA1 upregulation in mesangial cells exposed to high glucose levels (ESM Fig. 5a,b).

EGFR is activated in diabetic glomeruli Having shown that EGFR is transactivated by high glucose levels in mesangial cells, we wished to investigate whether this also occurs in vivo. We studied rats after 4 weeks of diabetes, with significant hyperglycaemia confirmed as 

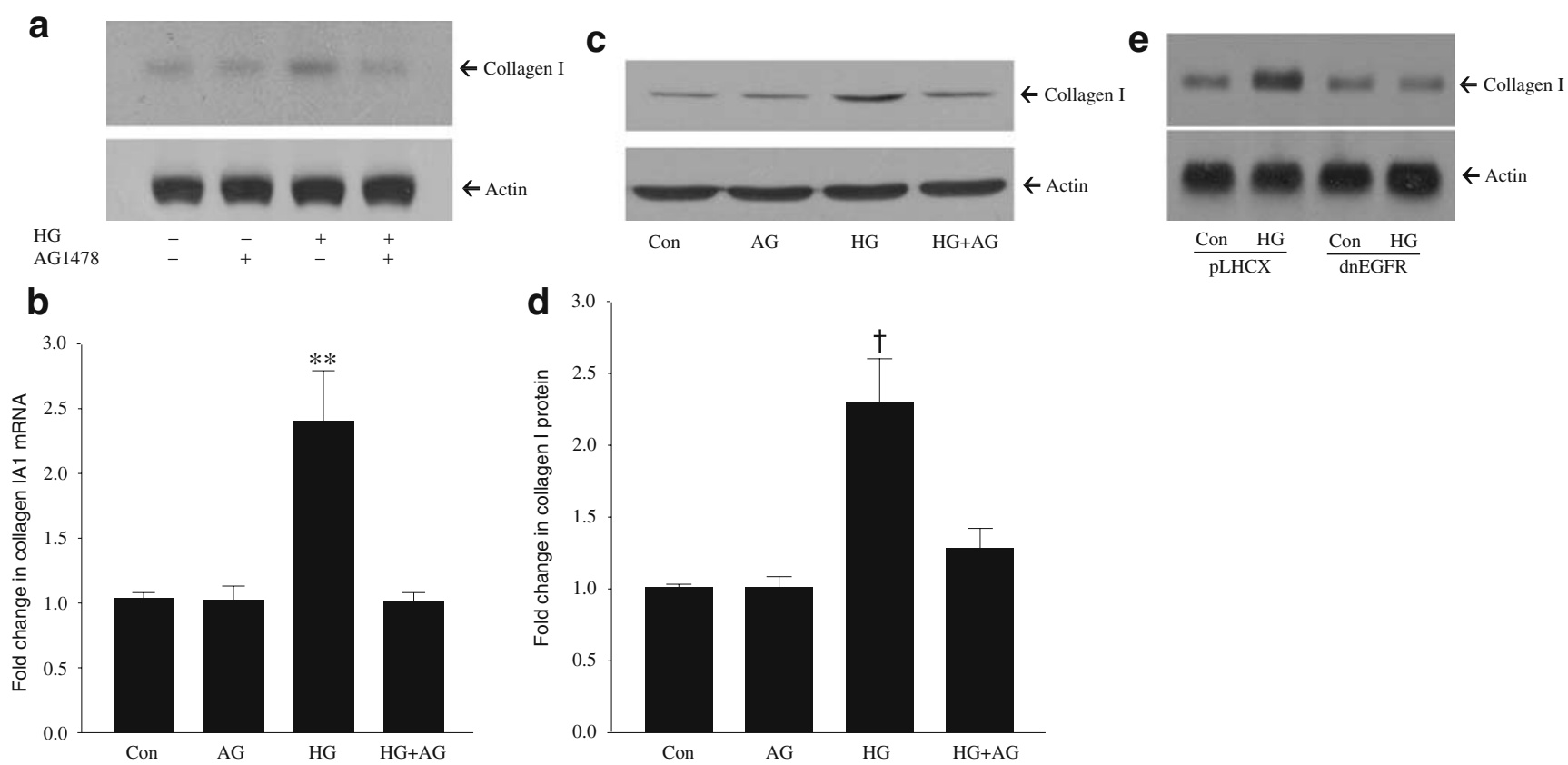

Fig. 6 EGFR inhibition prevents collagen I upregulation. a Northern blot analysis showing that high glucose $(H G)$-induced collagen IA1 transcript upregulation at 3 days was prevented by EGFR inhibition with AG1478 (AG; $5 \mu \mathrm{mol} / \mathrm{l}$ ), with results quantified in bar graph (b), ${ }^{* *} p<0.01$ vs all, $n=3$. c Immunoblot showing that the increase in

collagen I protein was also abrogated by EGFR blockade; results are quantified in bar graph (d), ${ }^{\dagger} p<0.02$ vs all others, $n=8$. e Northern blot showing that the high glucose level did not induce collagen I upregulation in cells overexpressing dominant-negative (dn) EGFR. Con control

identified above. Glomeruli were immunohistochemically identified by positive staining for von Willebrand factor (red) (Fig. 7a). EGFR Y1068 phosphorylation (green) is seen to be increased in glomeruli. Concordant immunoblotting of glomerular protein for EGFR Y1068 phosphorylation identified a clear increase in EGFR activation in diabetic glomeruli (Fig. 7b,c). We also sought to determine whether glomerular EFGR and collagen I co-localisation could be observed. ESM Fig. 6 shows that EGFR and collagen I co-localisation was observed in diabetic glomeruli.

\section{Discussion}

In this study, we observed induction of PI3K/Akt signalling in diabetic glomeruli and demonstrated that this pathway is an important novel mediator of collagen I production in mesangial cells exposed to a high glucose milieu. Further, we have identified that transactivation of the EGFR also occurs in vivo and is required for Akt activation and collagen I production. Pharmacological targeting of this pathway at multiple levels may provide a novel approach to treatment of diabetic renal disease.
Fig. 7 EGFR is activated in diabetic glomeruli. Diabetes was induced in rats by streptozotocin and tissue examined after 4 weeks of diabetes. a Immunofluorescence staining of cortex shows increased EGFR Y1068 phosphorylation (green) in diabetic glomeruli, identified by von Willebrand factor (red). b Immunoblot showing that increased EGFR Y1068 phosphorylation was also present in isolated glomeruli; results are quantified in bar graph (c), $\S_{p}<0.03, n=6$. Con control
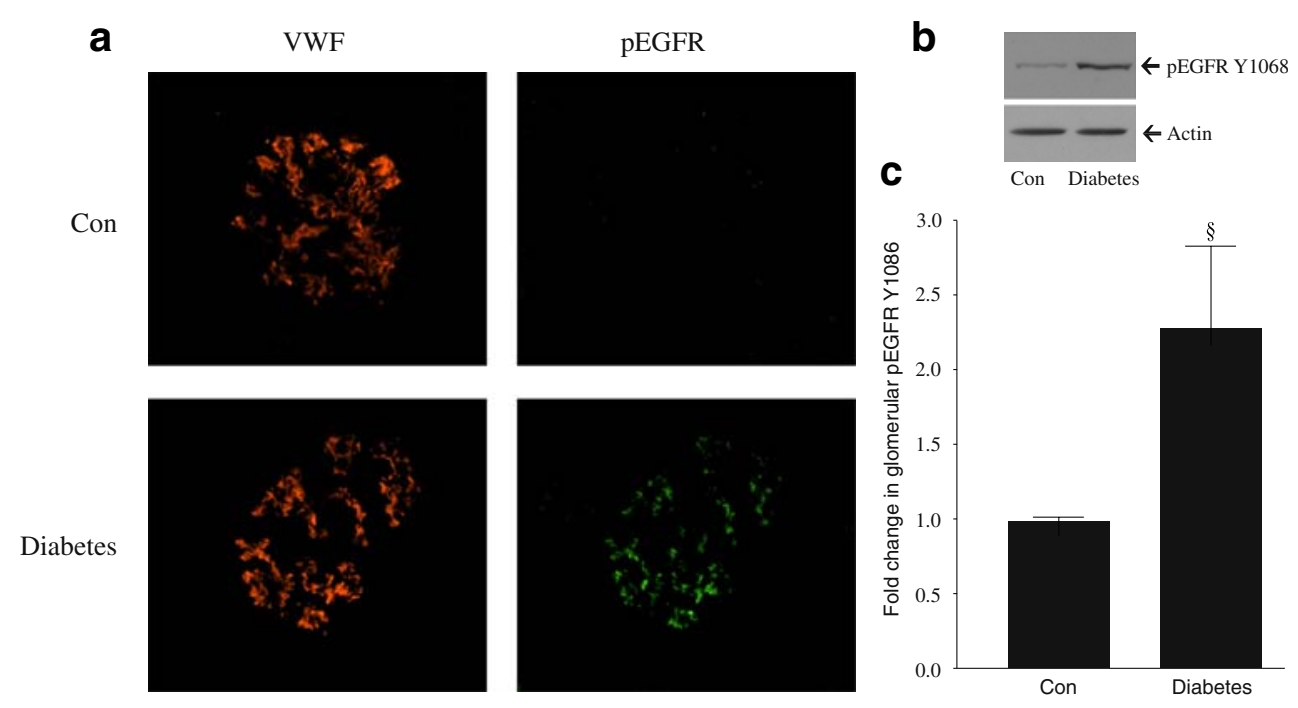
There is growing interest in the function of PI3K/Akt signalling in the setting of diabetes. In insulin-responsive tissue and cells, PI3K/Akt mediates insulin signalling through phosphorylation of the insulin receptor substrate 1 [26]. Insulin does not, however, regulate glucose uptake in mesangial cells. Recent studies have suggested that Akt might be activated by glucose in the absence of insulin. In mesangial cells in vitro, high glucose levels induced activation of PI3K and Akt, which then mediated cyclooxygenase-2 activation and cell proliferation [27]. In vivo, in the streptozotocin-induced rodent, an insulin-deficient model of diabetes, hyperglycaemia at both 3 days and 4 weeks increased Akt phosphorylation in brain [28]. Lloberas et al. [29] have recently shown increased phosphorylated Akt in whole kidney at 4 weeks of diabetes while increased levels of total and phosphorylated Akt were observed by others at 12 weeks in glomeruli [30]. Inhibition of PI3K reduced glomerular hypertrophy, an effect thought to be mediated through a decrease in downstream mammalian target of rapamycin (mTOR) activation [30]. Treatment with sirolimus, an inhibitor of mTOR, was also found to decrease cortical TGF- $\beta 1$ transcript upregulation in 4 week diabetic rats [29]. Our initial studies extended these findings, showing clear activation of Akt in glomeruli of insulin-deficient diabetic rats. Indeed, by immunoblotting, pAktS473 was significantly higher in glomerular preparations than in whole cortex, suggesting that the predominant site of activation was in glomeruli. Immunofluorescence confirmed these findings. Our subsequent studies confirmed that hyperglycaemia itself activates Akt in cultured primary mesangial cells, occurring early and with sustained effect, in keeping with our in vivo findings.

A role for Akt in matrix regulation has recently emerged. Overexpression of constitutively active Akt or the PI3K p1 $10 \alpha$ subunit in embryoid bodies resulted in massive collagen 4A1 and laminin accumulation [6]. Abnormal production of collagens 1, 2, 3 and laminin, as well as extracellular fibronectin assembly by keloid fibroblasts were blocked by LY294002 [31]; this role in fibronectin matrix assembly has also been seen in other cell types [32]. In hepatic stellate cells, basal collagen IA1 mRNA levels were decreased by LY294002 [7]. IL-13-induced collagen IA2 upregulation also depended on PI3K in dermal fibroblasts [33]. We have shown the importance of Akt activation in matrix generation in response to mechanical stress [5] and observed that overexpression of active Akt (AktDD) led to increased collagen I secretion by unstimulated mesangial cells [5]. Since glomerular matrix accumulation is the pathological hallmark of diabetic nephropathy, we assessed whether high glucose-induced Akt activation might be important for matrix upregulation. In the current study, we show that $\mathrm{PI} 3 \mathrm{~K} /$ Akt signalling is required for upregulation of collagen I by high glucose levels in mesangial cells.
The mechanisms by which PI3K/Akt may mediate matrix production are not yet known. We observed that high glucose-induced collagen I upregulation occurred at a transcriptional level and this corresponded with increased collagen I protein. The requirement for both PI3K and Akt was demonstrated using PI3K inhibitors, as well as a dominant-negative construct to inhibit Akt signalling. It has been suggested that PI3K signalling increases collagen transcript stability. In human lung fibroblasts, baseline collagen IA1 mRNA expression was inhibited by PI3K blockade with LY294002 [8]. Similarly, in hepatic stellate cells, LY294002 decreased basal collagen IA1 mRNA levels, although at a much slower rate (days vs hours) [7]. However, in human mesangial cells no effect of PI3K on basal collagen IA2 levels was found [9]. This is supported by our studies, in which neither wortmannin nor LY294002 affected the basal transcript levels of collagen IA1. This suggests that cell-specific factors affect the basal expression and involvement of PI3K/Akt in collagen synthesis.

Recently, TGF- $\beta 1$ has been shown to directly activate the $\mathrm{PI} 3 \mathrm{~K} /$ Akt pathway in some cells including mesangial cells, a process required for its induction of collagen synthesis $[8,9]$. Since TGF- $\beta 1$ is upregulated by high glucose levels in mesangial cells [34], it is possible that collagen upregulation in our studies might in part be due to autocrine activity of this cytokine. However, high glucose-induced Akt activation, particularly at early time points, cannot be ascribed to this mechanism. Alternatively, PI3K/Akt signalling might affect collagen I upregulation through direct transcriptional effects. The collagen IA1 promoter contains binding sites for numerous transcription factors, including activator protein 1 (AP-1) and $\mathrm{Sp} 1$ transcription factor (Sp1) [35]. Although these are not well-known downstream effectors of Akt, recently Akt was shown to regulate their activity. In endothelial cells, insulin-induced AP-1 activation was blocked by PI3K inhibition with wortmannin [36]. PI3K activation by asphalt fumes mediated AP-1 activation in epidermal cells [37]. Overexpression of a dominantnegative $\mathrm{p} 85$ construct in fibroblasts prevented growth factor-induced expression of c-fos, a component of the heterodimeric AP-1 [38]. Overexpression of constitutively active Akt has been shown to activate Sp1, while upregulation of vascular endothelial growth factor by Akt is mediated through $\mathrm{Sp} 1$ [39]. The transcriptional activity of both AP-1 and Sp1 was shown to be upregulated by high glucose levels in mesangial cells [40, 41]. Further studies are needed to establish whether these or other transcription factors might play a role in PI3K/Akt-mediated collagen IA1 upregulation by high glucose levels.

Although growth factor-induced PI3K/Akt activation through cognate receptors is well-defined [26], it is not known how high glucose levels might activate this signalling pathway. PI3K is composed of a catalytic 
(p110) and regulatory subunits (p85, p101) [11]. Binding of the regulatory subunit to a phosphotyrosine residue, such as found on activated growth factors, is required for release of autoinhibition of its catalytic subunit [11]. Since EGFR is known to activate PI3K/Akt and its transactivation serves in signal transduction for diverse non-ligand-mediated stimuli $[10,14]$, we assessed whether this might also be the case in high glucose-mediated PI3K activation. Indeed, we found the EGFR to be activated by high glucose levels and this transactivation was shown to be required for downstream Akt activation and collagen I synthesis in mesangial cells by use of both a specific tyrphostin inhibitor and a dominant-negative construct. Interestingly, EGF upregulation has been observed in the streptozotocin diabetes model, with increased EGF transcript upregulation and urinary excretion as early as 1 day after streptozotocin injection [42]. Although expression has been noted only in tubular segments, EGFR inhibition was able to reduce glomerular hypertrophy after 3 weeks of streptozotocin-induced diabetes [43]. In this study, we showed that EGFR and collagen I co-localisation was increased in glomeruli and tubular areas in diabetic animals. In vitro, high glucose levels have been shown to increase EGFR phosphorylation in proximal tubular epithelial cells [44]. In vascular smooth muscle cells, angiotensin II-induced Akt activation was augmented by high glucose levels. This was shown to be facilitated by increased responsiveness of an $N$-glycosylated EGFR in a hyperglycaemic environment [24]. While longer-term responses to high glucose levels such as matrix induction may invoke secondary pathways such as autocrine angiotensin II signalling, this is unlikely to explain our findings of short-term (minute) activation of Akt by high glucose levels. This study is thus the first to show EGFR transactivation by high glucose levels in resident glomerular cells, its involvement in matrix gene upregulation, as well as EGFR activation in vivo in diabetic glomeruli. However, the mechanism of transactivation of this receptor by high glucose levels is unknown.

Here, we have identified the PI3K/Akt signalling pathway as an important novel mediator of collagen I production by mesangial cells exposed to high glucose levels and have also confirmed that Akt is activated in the glomeruli of insulin-deficient diabetic animals. Further, we have identified that transactivation of the EGFR is required for Akt activation. Current treatment options are not effective in fully preventing the progression of diabetic kidney disease. Further long-term studies are required to pursue the effects of blockade of EGFR/PI3K/Akt signalling on renal survival in diabetes. However, our study suggests that pharmacological targeting of this pathway at single or multiple levels may provide a novel approach to the treatment of diabetic renal disease.
Acknowledgements We are grateful for the support of the Canadian Institutes of Health Research and Canadian Diabetes Association (J. Krepinsky). D. Wu is a recipient of the Krescent Fellowship sponsored by the Kidney Foundation of Canada and F. Peng is a recipient of the Father Sean O'Sullivan Research Center Fellowship. We thank J. Woodgett (Samuel Lunenfeld Research Institute, University of Toronto, Toronto, ON, Canada) for kindly providing pcDNA3 HAAktAAA and S. Parsons for providing pcDNA3 EGFR K721A (University of Virginia Health System, Charlottesville, VA, USA).

Duality of interest The authors declare that there is no duality of interest associated with this manuscript.

\section{References}

1. Marks JB, Raskin P (1998) Nephropathy and hypertension in diabetes. Med Clin North Am 82:877-907

2. No authors listed (2000) Retinopathy and nephropathy in patients with type 1 diabetes four years after a trial of intensive therapy. The Diabetes Control and Complications Trial/Epidemiology of Diabetes Interventions and Complications Research Group. N Engl J Med 342:381-389

3. Lewis EJ, Hunsicker LG, Bain RP, Rohde RD (1993) The effect of angiotensin-converting-enzyme inhibition on diabetic nephropathy. The Collaborative Study Group. N Engl J Med 329: 1456-1462

4. Mason RM, Wahab NA (2003) Extracellular matrix metabolism in diabetic nephropathy. J Am Soc Nephrol 14:1358-1373

5. Krepinsky JC, Li Y, Chang Y et al (2005) Akt mediates mechanical strain-induced collagen production by mesangial cells. J Am Soc Nephrol 16:1661-1672

6. Li X, Talts U, Talts JF, Arman E, Ekblom P, Lonai P (2001) Akt/ $\mathrm{PKB}$ regulates laminin and collagen IV isotypes of the basement membrane. Proc Natl Acad Sci USA 98:14416-14421

7. Reif S, Lang A, Lindquist JN et al (2003) The role of focal adhesion kinase-phosphatidylinositol 3-kinase-akt signaling in hepatic stellate cell proliferation and type I collagen expression. J Biol Chem 278:8083-8090

8. Ricupero DA, Poliks CF, Rishikof DC, Cuttle KA, Kuang PP, Goldstein RH (2001) Phosphatidylinositol 3-kinase-dependent stabilization of alpha1(I) collagen mRNA in human lung fibroblasts. Am J Physiol Cell Physiol 281:C99-C105

9. Runyan CE, Schnaper HW, Poncelet AC (2004) The phosphatidylinositol 3-kinase/Akt pathway enhances Smad3-stimulated mesangial cell collagen I expression in response to transforming growth factor-beta1. J Biol Chem 279:2632-2639

10. Brazil DP, Hemmings BA (2001) Ten years of protein kinase B signalling: a hard Akt to follow. Trends Biochem Sci 26:657-664

11. Cantley LC (2002) The phosphoinositide 3-kinase pathway. Science 296:1655-1657

12. Scheid MP, Woodgett JR (2001) PKB/AKT: functional insights from genetic models. Nat Rev Mol Cell Biol 2:760-768

13. Chan TO, Rittenhouse SE, Tsichlis PN (1999) AKT/PKB and other D3 phosphoinositide-regulated kinases: kinase activation by phosphoinositide-dependent phosphorylation. Annu Rev Biochem 68:965-1014

14. Zwick E, Hackel PO, Prenzel N, Ullrich A (1999) The EGF receptor as central transducer of heterologous signalling systems. Trends Pharmacol Sci 20:408-412 
15. Krepinsky JC, Ingram AJ, Tang D, Wu D, Liu L, Scholey JW (2003) Nitric oxide inhibits stretch-induced MAPK activation in mesangial cells through RhoA inactivation. J Am Soc Nephrol $14: 2790-2800$

16. Wang Q, Somwar R, Bilan PJ et al (1999) Protein kinase B/Akt participates in GLUT4 translocation by insulin in L6 myoblasts. Mol Cell Biol 19:4008-4018

17. Fierlbeck W, Liu A, Coyle R, Ballermann BJ (2003) Endothelial cell apoptosis during glomerular capillary lumen formation in vivo. J Am Soc Nephrol 14:1349-1354

18. Bellacosa A, Chan TO, Ahmed NN et al (1998) Akt activation by growth factors is a multiple-step process: the role of the $\mathrm{PH}$ domain. Oncogene 17:313-325

19. Nicholson KM, Anderson NG (2002) The protein kinase B/Akt signalling pathway in human malignancy. Cell Signal 14:381-395

20. Moriguchi Y, Matsubara H, Mori Y et al (1999) Angiotensin IIinduced transactivation of epidermal growth factor receptor regulates fibronectin and transforming growth factor-beta synthesis via transcriptional and posttranscriptional mechanisms. Circ Res 84:1073-1084

21. Downward J, Parker P, Waterfield MD (1984) Autophosphorylation sites on the epidermal growth factor receptor. Nature 311:483-485

22. Rice AB, Moomaw CR, Morgan DL, Bonner JC (1999) Specific inhibitors of platelet-derived growth factor or epidermal growth factor receptor tyrosine kinase reduce pulmonary fibrosis in rats. Am J Pathol 155:213-221

23. Ingram AJ, Ly H, Thai K, Kang MJ, Scholey JW (1999) Mesangial cell signaling cascades in response to mechanical strain and glucose. Kidney Int 56:1721-1728

24. Konishi A, Berk BC (2003) Epidermal growth factor receptor transactivation is regulated by glucose in vascular smooth muscle cells. J Biol Chem 278:35049-35056

25. Saito Y, Haendeler J, Hojo Y, Yamamoto K, Berk BC (2001) Receptor heterodimerization: essential mechanism for plateletderived growth factor-induced epidermal growth factor receptor transactivation. Mol Cell Biol 21:6387-6394

26. Lawlor MA, Alessi DR (2001) PKB/Akt: a key mediator of cell proliferation, survival and insulin responses? J Cell Sci 114: 2903-2910

27. Sheu ML, Ho FM, Chao KF, Kuo ML, Liu SH (2004) Activation of phosphoinositide 3-kinase in response to high glucose leads to regulation of reactive oxygen species-related nuclear factorkappaB activation and cyclooxygenase- 2 expression in mesangial cells. Mol Pharmacol 66:187-196

28. Clodfelder-Miller B, De Sarno P, Zmijewska AA, Song L, Jope RS (2005) Physiological and pathological changes in glucose regulate brain akt and glycogen synthase kinase-3. J Biol Chem 280:39723-39731

29. Lloberas N, Cruzado JM, Franquesa M et al (2006) Mammalian target of rapamycin pathway blockade slows progression of diabetic kidney disease in rats. J Am Soc Nephrol 17:1395-1404
30. Nagai K, Matsubara T, Mima A et al (2005) Gas6 induces Akt/ mTOR-mediated mesangial hypertrophy in diabetic nephropathy. Kidney Int 68:552-561

31. Lim IJ, Phan TT, Tan EK et al (2003) Synchronous activation of ERK and phosphatidylinositol 3-kinase pathways is required for collagen and extracellular matrix production in keloids. J Biol Chem 278:40851-40858

32. Wierzbicka-Patynowski I, Schwarzbauer JE (2002) Regulatory role for SRC and phosphatidylinositol 3-kinase in initiation of fibronectin matrix assembly. J Biol Chem 277:19703-19708

33. Jinnin M, Ihn H, Yamane K, Tamaki K (2004) Interleukin-13 stimulates the transcription of the human alpha2(I) collagen gene in human dermal fibroblasts. J Biol Chem 279:41783-41791

34. Weigert C, Sauer U, Brodbeck K, Pfeiffer A, Haring HU, Schleicher ED (2000) AP-1 proteins mediate hyperglycemia-induced activation of the human TGF-beta1 promoter in mesangial cells. J Am Soc Nephrol 11:2007-2016

35. Gao CF, Wang H, Wang AH, Wan WD, Wu YA, Kong XT (2004) Transcriptional regulation of human alpha1(I) procollagen gene in dermal fibroblasts. World J Gastroenterol 10:1447-1451

36. Fisslthaler B, Benzing T, Busse R, Fleming I (2003) Insulin enhances the expression of the endothelial nitric oxide synthase in native endothelial cells: a dual role for Akt and AP-1. Nitric Oxide $8: 253-261$

37. Ma C, Wang J, Luo J (2003) Exposure to asphalt fumes activates activator protein-1 through the phosphatidylinositol 3-kinase/Akt signaling pathway in mouse epidermal cells. J Biol Chem 278:44265-44272

38. Jhun BH, Rose DW, Seely BL et al (1994) Microinjection of the SH2 domain of the 85-kilodalton subunit of phosphatidylinositol 3-kinase inhibits insulin-induced DNA synthesis and c-fos expression. Mol Cell Biol 14:7466-7475

39. Pore N, Liu S, Shu HK et al (2004) Sp1 is involved in Akt-mediated induction of VEGF expression through an HIF-1-independent mechanism. Mol Biol Cell 15:4841-4853

40. Wilmer WA, Cosio FG (1998) DNA binding of activator protein-1 is increased in human mesangial cells cultured in high glucose concentrations. Kidney Int 53:1172-1181

41. Goldberg HJ, Whiteside CI, Fantus IG (2002) The hexosamine pathway regulates the plasminogen activator inhibitor-1 gene promoter and $\mathrm{Sp} 1$ transcriptional activation through protein kinase C-beta I and -delta. J Biol Chem 277:33833-33841

42. Gilbert RE, Cox A, McNally PG et al (1997) Increased epidermal growth factor in experimental diabetes related kidney growth in rats. Diabetologia 40:778-785

43. Wassef L, Kelly DJ, Gilbert RE (2004) Epidermal growth factor receptor inhibition attenuates early kidney enlargement in experimental diabetes. Kidney Int 66:1805-1814

44. Saad S, Stevens VA, Wassef L et al (2005) High glucose transactivates the EGF receptor and up-regulates serum glucocorticoid kinase in the proximal tubule. Kidney Int 68:985-997 